\title{
Identification and distribution of a single nucleotide polymorphism responsible for the catechin content in tea plants
}

\author{
Chen-Kai Jiang ${ }^{1,2}$, Jian-Qiang Ma', Yu-Fei Liư ${ }^{1,3}$, Jie-Dan Chen $\mathbb{1}^{1}$, De-Jiang $\mathrm{Ni}^{2}$ and Liang Chen (1)
}

\begin{abstract}
Catechins are the predominant products in tea plants and have essential functions for both plants and humans. Several genes encoding the enzymes regulating catechin biosynthesis have been identified, and the identification of single nucleotide polymorphisms (SNPs) resulting in nonsynonymous mutations within these genes can be used to establish a functional link to catechin content. Therefore, the transcriptomes of two parents and four filial offspring were sequenced using next-generation sequencing technology and aligned to the reference genome to enable SNP mining. Subsequently, 176 tea plant accessions were genotyped based on candidate SNPs using kompetitive allelespecific polymerase chain reaction (KASP). The catechin contents of these samples were characterized by highperformance liquid chromatography (HPLC), and analysis of variance (ANOVA) was subsequently performed to determine the relationship between genotypes and catechin content. As a result of these efforts, a SNP within the chalcone synthase (CHS) gene was shown to be functionally associated with catechin content. Furthermore, the geographical and interspecific distribution of this SNP was investigated. Collectively, these results will contribute to the early evaluation of tea plants and serve as a rapid tool for accelerating targeted efforts in tea breeding.
\end{abstract}

\section{Introduction}

Tea, an important healthy beverage that is popular worldwide, is made from fresh leaves harvested from tea plants belonging to the family Theaceae, genus Camellia L., section Thea (L.) Dyer, including Camellia sinensis (L.) O. Kuntze, with the varieties var. sinensis, var. assamica (Masters) Kitamura, and var. pubilimba Chang and the related wild species C. tachangensis F. C. Zhang, C. taliensis (W. W. Smith) Melchior, C. crassicolumna Chang, and C. gymnogyna Chang ${ }^{1}$. Among them, C. sinensis, the main source of commercially grown tea, is

\footnotetext{
Correspondence: De-Jiang Ni (nidj@mail.hzau.edu.cn) or

Liang Chen (liangchen@tricaas.com)

${ }^{1}$ Key Laboratory of Tea Biology and Resources Utilization, Ministry of Agriculture and Rural Affairs, Tea Research Institute of the Chinese Academy of Agricultural Sciences, 9 South Meiling Road, Hangzhou, Zhejiang 310008, China

${ }^{2}$ College of Horticulture and Forestry Science, Huazhong Agricultural University, 1 Shizishan Street, Hongshan District, Wuhan, Hubei 430070, China Full list of author information is available at the end of the article.
}

widely cultivated in more than 50 countries $^{2}$. The most extensive genetic variation of tea germplasm worldwide is found in China due to its long history of tea breeding and substantial cultivated genetic resources ${ }^{3}$. These vast genetic resources can serve as an invaluable foundation for modern tea breeding endeavors, and efforts should be taken to ensure that the germplasm is conserved in the future. Currently, the primary objectives for tea breeding are aimed toward the management of early flushing of growth in the spring, special characteristics, high yield, and increased resistance to biotic and abiotic stresses ${ }^{4}$.

Conventional tea breeding is a slow and expensive process owing to the characteristically long juvenile period of tea plants. Typically, at least a 20 -year time period is required to complete a cycle of tea breeding, starting from mass selection to local adaptability testing and final release to the public ${ }^{5}$. To date, individual selection and controlled hybridization represent traditional breeding approaches for tea plants. Therefore, novel methods to effectively evaluate 
and select desirable alleles from existing tea resources are urgently needed to improve the efficiency of tea breeding. Over the last decade, mutation breeding and molecular assisted selection have been further developed with the application of new technologies, especially those related to molecular markers, including restriction fragment length polymorphism (RFLP), amplified fragment length polymorphism (AFLP), simple sequence repeat (SSR), and single nucleotide polymorphism (SNP) analyses. Collectively, these techniques help to efficiently identify and isolate functional sequences with the goal of improving the precision of breeding ${ }^{6-9}$. As described by Lander in $1996^{10}$, SNPs exhibit a typical mutation rate of $\sim 10^{-9}$ per nucleotide and have received increasing attention from researchers for their critical role in allele mining, genetic mapping and germplasm identification. Nevertheless, most of the SNPs identified in tea plant are putative ${ }^{11,12}$ and have not been functionally assessed. Specifically, only a few SNPs have been validated and developed into markers for screening tea genetic resources to date.

Flavonoids and their derivatives are a major group of compounds in tea ${ }^{13}$ that are known to confer stress tolerance to tea plants during growth and development ${ }^{14,15}$. Polyphenols are the major flavonoids found within fresh and commercial tea leaves ${ }^{16}$. Catechins, which account for more than $70 \%$ of polyphenols ${ }^{17}$, can be divided into ester and nonester catechins ${ }^{18}$. These multifunctional polyphenols help to reduce reactive oxygen species and improve the environmental adaptability of plants ${ }^{19,20}$. In addition, they possess beneficial functions related to the improvement of cardiac function and have anti-inflammatory, antiaging and lipid-lowering effects ${ }^{21-23}$. In tea, the main catechins are epigallocatechin gallate (EGCG), epicatechin gallate (ECG), epigallocatechin (EGC), and epicatechin $(E C)^{18}$. Some key genes in the catechin biosynthesis pathway, including phenylalanine ammonia-lyase $(P A L)$, cinnamate 4-hydroxylase $(C 4 H)$, 4-coumarate-CoA ligase $(4 C L)$, chalcone synthase $(C H S)$, chalcone isomerase $(\mathrm{CHI})$, flavonoid $3^{\prime}$-hydroxylase $\left(F 3^{\prime} H\right)$, flavonoid $3^{\prime} 5^{\prime}$-hydroxylase $\left(F 3^{\prime} 5^{\prime} H\right)$, dihydroflavonol 4-reductase $(D F R)$, flavonol synthase $(F L S)$, anthocyanidin reductase $(A N R)$, and leucoanthocyanidin reductase $(L A R)$, have been identified and cloned in tea plants ${ }^{24}$. Alterations in the expression level and mutations of these gene sequences, such as base substitutions, insertions, deletions, and dynamic mutations, affect compound metabolism ${ }^{25,26}$. Regarding the effects on catechin regulation at the gene level, very limited information exists pertaining to the effects of SNPs within gene sequences ${ }^{27,28}$. In the current study, two parents were artificially hybridized to obtain the first filial (F1) offspring. The transcriptomes from two parents and four selected F1 offspring with significant differences in catechins were sequenced and aligned to the reference genome. Twenty-eight candidate SNPs within coding sequences of the highly expressed genes regulating flavonoids were selected for kompetitive allele-specific polymerase chain reaction (KASP). This technical approach aims to identify the SNPs responsible for catechin content. Furthermore, KASP provides insights into the development of SNP markers for the evaluation and selection of unique tea resources, which can contribute to the effective management and genetic improvement of tea plants.

\section{Results}

\section{Sequencing and mapping}

The cDNA libraries were constructed and sequenced by using an Illumina HiSeq 2500 platform. Specifically, transcriptome sequencing data were generated from six samples: 'Yingshuang' (YS), 'Beiyue Danzhu' (BD), F1-1, F1-2, F1-3, and F1-4. Within the generated datasets, the raw reads ranged from 29.19 to 100.81 million reads, with $>97 \%$ of the reads passing the quality and trimming filters as described in Table 1. Subsequently, the clean reads were aligned with two published genomes of tea plant, namely, C. sinensis var. sinensis (CSS) ${ }^{29}$ and C. sinensis var. assamica $(\mathrm{CSA})^{30}$. The ratio of total mapped reads between each of the six samples and CSS or CSA was $90.77 \sim 92.40 \%$ and $83.00 \sim 87.40 \%$, respectively. The mapping information demonstrated that the genetic relationship between the six samples and CSS was more similar than that with CSA. Therefore, the following

\section{Table 1 Description of the RNA sequencing data and} mapping information

\begin{tabular}{lccccccc}
\hline Sample & YS & BD & F1-1 & F1-2 & F1-3 & F1-4 \\
\hline Raw reads $\left(\times 10^{6}\right)$ & & 29.19 & 105.29 & 90.04 & 85.57 & 80.70 & 100.81 \\
Clean reads & & 98.39 & 98.65 & 97.58 & 97.52 & 97.36 & 97.73 \\
percentage (\%) & & & & & & & \\
Q20 (\%) & & 95.67 & 95.51 & 94.52 & 94.56 & 94.36 & 94.61 \\
Q30 (\%) & & 92.00 & 91.73 & 89.52 & 89.63 & 89.27 & 89.69 \\
GC content (\%) & & 46.29 & 46.33 & 45.02 & 44.95 & 45.65 & 45.23 \\
Total mapped (\%) & CSS & 90.77 & 91.12 & 92.30 & 92.13 & 92.40 & 91.78 \\
& CSA & 83.00 & 86.52 & 86.45 & 87.32 & 87.40 & 86.86 \\
Multiple mapped (\%) & CSS & 7.33 & 5.73 & 5.69 & 5.38 & 5.44 & 5.47 \\
& CSA & 3.39 & 3.28 & 3.35 & 3.36 & 3.40 & 3.39 \\
Uniquely & CSS & 83.43 & 85.39 & 86.61 & 86.75 & 86.95 & 86.31 \\
mapped (\%) & CSA & 79.62 & 83.24 & 83.11 & 83.96 & 84.00 & 83.47 \\
Nonsplice reads (\%) & CSS & 56.67 & 55.86 & 57.02 & 56.58 & 55.99 & 55.57 \\
& CSA & 54.60 & 55.25 & 55.41 & 55.47 & 54.79 & 54.49 \\
Splice reads (\%) & CSS & 26.76 & 29.52 & 29.59 & 30.18 & 30.97 & 30.74 \\
& CSA & 25.02 & 27.99 & 27.70 & 28.48 & 29.21 & 28.97 \\
\hline
\end{tabular}

${ }^{\mathrm{a}}$ and ${ }^{\mathrm{b}}$ represent the percentages of correct base identification that were $>99 \%$ and $99.9 \%$, respectively 
analysis was based on the sequences that were mapped to the CSS genome.

\section{Analysis of the gene expression levels}

Twelve genes that were functionally related to metabolism were randomly chosen for quantitative real-time polymerase chain reaction (qRT-PCR) analysis, and their primers are listed in Table S1. The FPKM values were calculated to determine the expression levels based on the number of mapped genes. Collectively, these data revealed that the RNA sequencing results were generally consistent with the qRT-PCR data presented in Fig. 1.

\section{KEGG enrichment analysis of the DEGs}

Differentially expressed genes (DEGs) were identified by comparative analyses between every two samples. Then, a KEGG enrichment analysis was conducted to investigate the functional roles of the identified DEGs. Figure 2 illustrates the top 20 pathway enrichment items of the parents BD and YS, where a decrease in the $Q$ value is directly related to an increased significance of the enrichment. Consequently, "flavonoid biosynthesis" and "alpha-linolenic acid metabolism" were the most notable enriched pathways, with the top two groups of genes being involved with metabolic pathways and the biosynthesis of secondary metabolites. Among these pathways, the flavonoid and phenylpropanoid biosynthetic pathways were of interest for further analysis since catechins are products of phenylpropanoid and flavonoid metabolism. Among the pathways for the upregulated DEGs from the F1-2 vs. BD comparison, "flavonoid biosynthesis" was the most enriched pathway (Fig. S1), whereas "phenylpropanoid biosynthesis" was the most enriched pathway among the pathways for the

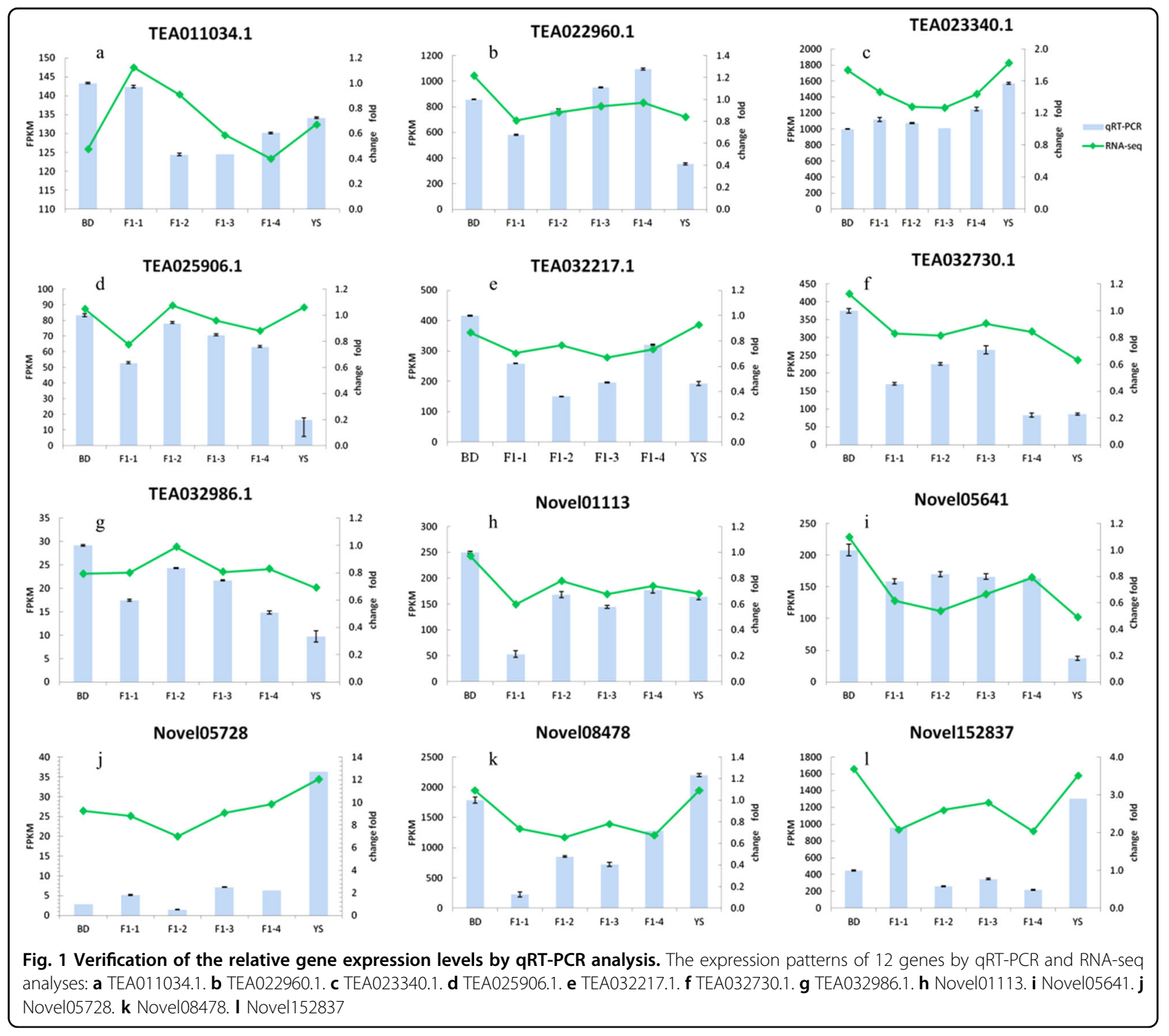




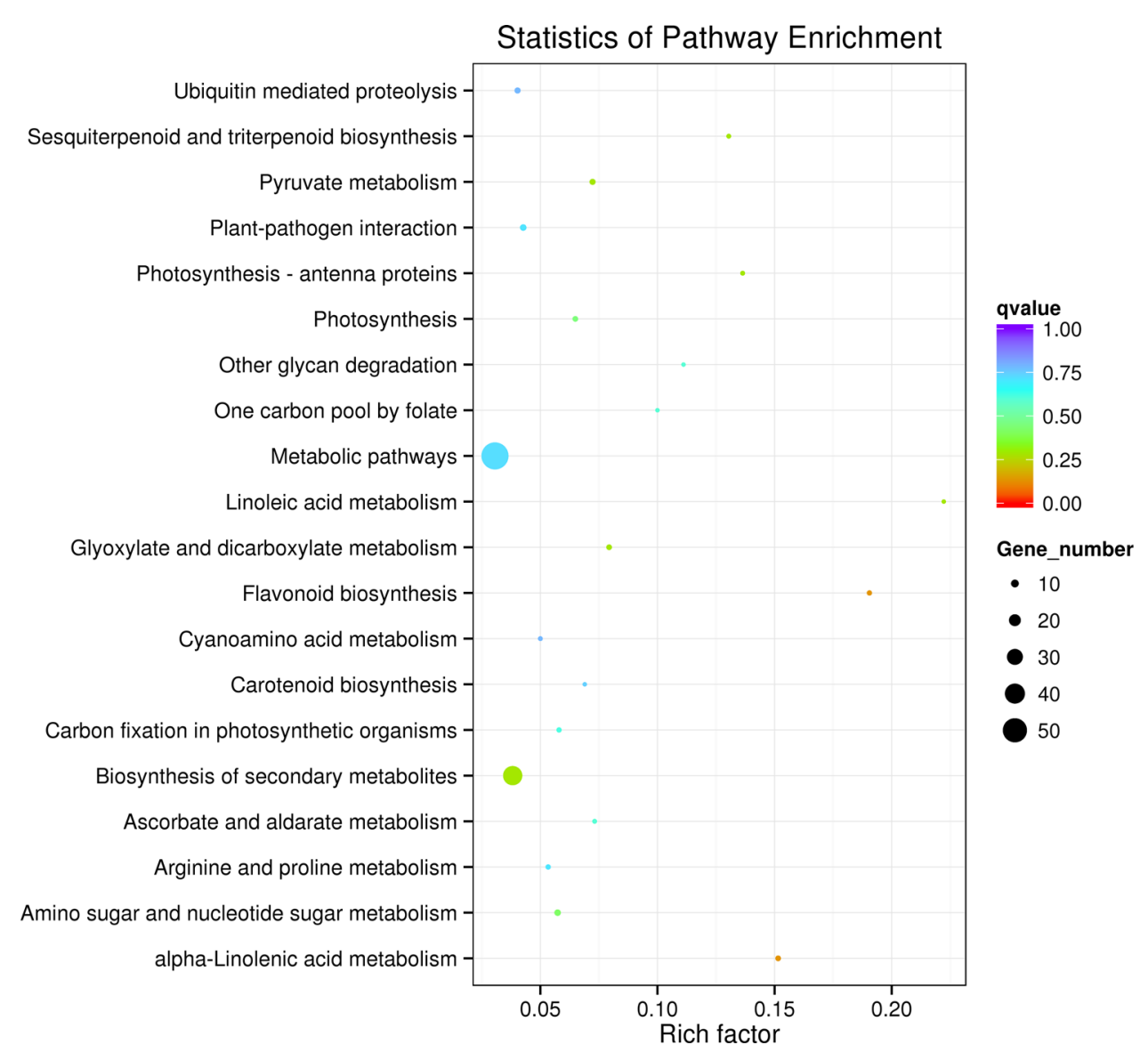

Fig. 2 Top 20 enriched KEGG pathways between BD and YS

upregulated DEGs from the $\mathrm{BD}$ vs. YS comparison (Fig. S2).

\section{Detection and functional validation of the SNPs}

The discovery and identification of the SNPs was performed according to the CSS genome. By using a combined analysis of six transcriptomes and a reference genome, we identified a total of $1,376,014$ high-quality putative SNPs. In addition to the aforementioned DEGs from the flavonoid and phenylpropanoid biosynthetic pathways, eleven major genes ( $P A L, C 4 H, 4 C L, C H S, C H I$, $\left.F 3^{\prime} H, F 3^{\prime} 5^{\prime} H, D F R, F L S, A N R, L A R\right)$ that are associated with flavonoid and phenylpropanoid metabolism on NCBI (https://www.ncbi.nlm.nih.gov/) were identified and aligned to the gene sequences obtained from RNA sequencing data. Then, the SNPs identified within the coding sequences of these genes were further studied by KASP analysis. Twenty accessions were tested two times to examine the reliability of the SNP identification (Table S2). Among the accessions, only one was inconsistent, demonstrating that $95 \%$ of the genotypes were reliable. Eventually, SNP556781 was validated and found to regulate the total catechin content (TCC) in tea plants, with three specific genotypes existing (AA, AG, and GG) (Fig. 3a, Tables S2 and S5).

Specifically, a nonsynonymous SNP is located in the coding sequence of TEA023340.1 where the 43rd amino acid is isoleucine (Ile) or threonine (Thr) when A or G is located at the SNP556781 site (Fig. S3a), respectively. The genotype AA only accounted for $6.86 \%$, whereas the GG genotype accounted for up to $64.00 \%$. Thus, we concluded that GG was the dominant genotype at the SNP556781 site.

As shown in Fig. 3b, SNP556781 makes a major contribution to TCC $(p<0.01)$ based on ANOVA (Table S3). The median TCC values of the tea accessions with the genotypes AA, AG, and GG were $16.59 \%, 14.55 \%$, and $14.41 \%$, respectively. Collectively, these data suggest that the tea plants with the homozygous genotype AA could accumulate substantially more catechin than those with the homozygous genotype GG and heterozygous genotype AG. However, no significant difference in TCC was observed between genotypes AG and GG.

\section{Genotype distribution of SNP556781}

As shown in Fig. 4a, genotype AA only appears in Yunnan, which was the origin center of the tea plant ${ }^{31}$, 


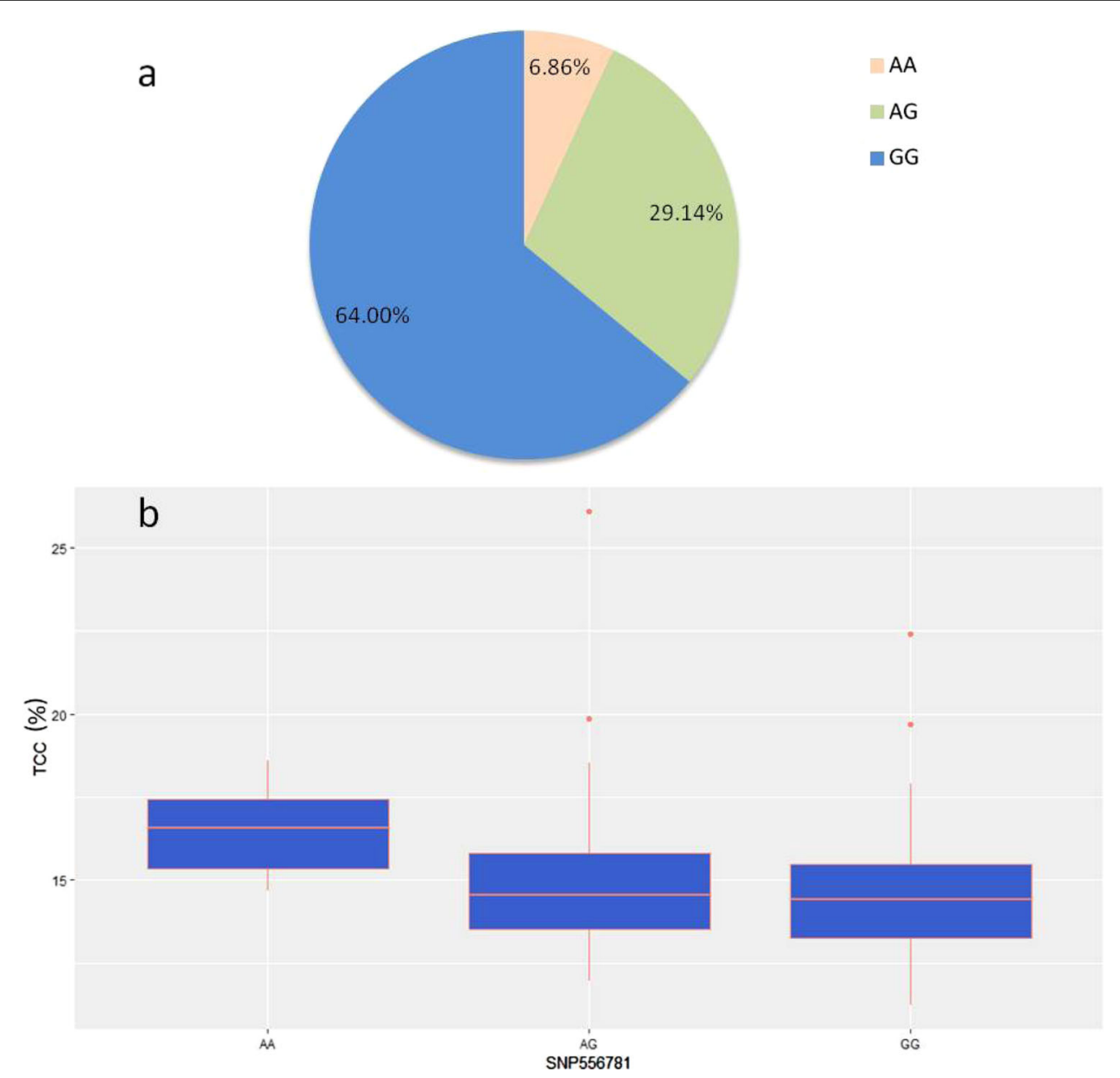

Fig. 3 Genotypes of SNP556781 in 176 accessions of tea plants. a The percentages of AA, AG, and GG. b Total catechin content in tea plants with three different SNP556781 genotypes

implying that AA was the primitive genotype. Tea plants from Yunnan, Guizhou, Guangxi, Hunan, Guangdong, Anhui, Zhejiang, and Jiangsu possessed the genotype AG. A distinctive genotype constitution was observed in a different group of Thea sections (Fig. 4b). Specifically, $C$. taliensis, $C$. sinensis var. sinensis, and $C$. sinensis var. assamica had three genotypes. Nevertheless, it was difficult to determine whether the remaining groups lacked one or two genotypes, since insufficient samples were available for the analysis, with the exception of $C$. sinensis var. sinensis. In addition, all C. sinensis var. sinensis cultivars with the AA genotype are F1 individuals that are the offspring of YS and BD.

\section{Discussion}

SNPs are becoming an increasingly popular and powerful tool to enable a genetic analysis of the functional mechanisms in tea plants, and research on this topic has resulted in significant achievements in the last 5 years. For example, large-scale SNPs combined with SSRs in an F1 population $(\mathrm{YS} \times \mathrm{BD})$ were used for the construction of the first high-density genetic map of tea plant in $2015^{32}$. Then, a high-density SNP linkage map was constructed, and QTLs related to flavonoids that were dependent on the F1 population (LJ43 $\times$ BHZ) were identified in $2018^{12}$. Candidate QTLs and proteins were shown to be involved in flavonoid biosynthesis using DArTseq markers via two F1 populations (TRFK $303 / 577 \times \mathrm{GW}$ Ejulu and GW Ejulu $\times$ TRFK 303/577) in 2018 and $2019^{33,34}$. SNPs and InDels were identified based on whole genome sequences of 'Shuchazao' and 'Yunkang 10' in 2019. Many of these SNPs/InDels were in catechin/caffeine biosynthesisrelated genes, although they have not been validated ${ }^{35}$.

Although some QTLs related to flavonoids were identified in these previous studies, they have not been further validated. As a result, it is still difficult to take full advantage of the putative QTLs in molecular markerassisted breeding efforts. In the present study, we aimed to identify novel SNPs by adopting a new strategy. First, precise information to enable SNP discovery was obtained 


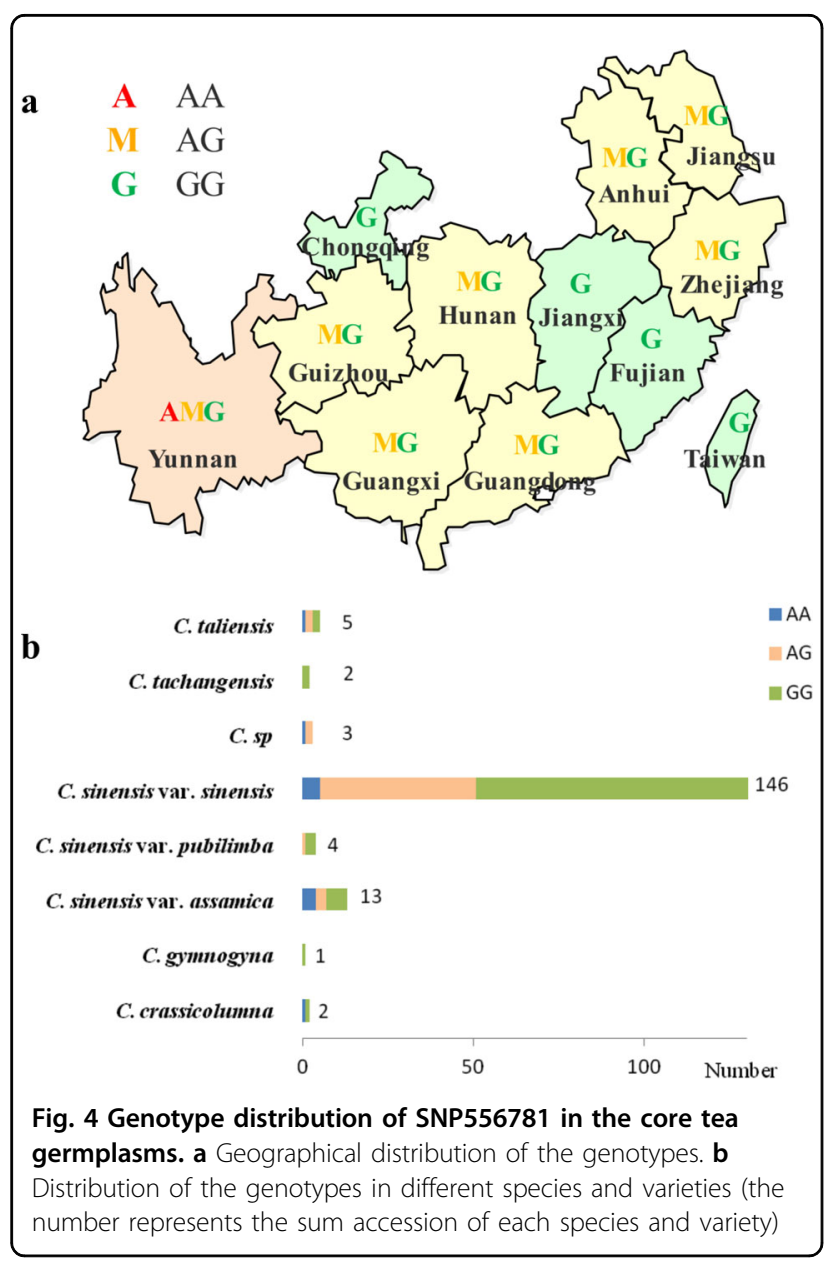

by using the reference genomes of tea plant. Second, candidate SNPs selected from RNA sequencing data obtained from test groups were further explored by KASP. Finally, the verification group was more representative and comprehensive than the previous group since it contained natural populations in addition to an F1 population. Collectively, the results showed that this strategy is an economic and effective way to mine SNPs that are functionally related to catechin content.

SNPs affect the functions of genes via nonsynonymous mutations or by regulating expression levels. The SNP position in the gene sequence controls its effect on TCC. For example, TEA023340.1 is located within scaffold2074: 55434-55909, which contains the sequence encoding CHS (EC 2.3.1.74). CHS is the rate-limiting enzyme of flavonoid biosynthesis in the general phenylpropanoid pathway $^{36}$. Specifically, CHS is the first committed enzyme of flavonoid biosynthesis and catalyzes the stepwise condensation of three acetate residues from malonyl-CoA with the phenylpropanoid biosynthetic intermediate $\mathrm{p}$ coumaryl $\mathrm{CoA}$ to form naringenin chalcone, which leads to the synthesis of various flavonoid derivatives ${ }^{37}$. By using a BLAST analysis in the Protein Data Bank, $C H S$ in
C. sinensis was shown to be most similar to chain A of CHS (PDB ID: 5CU5) in apple, with a similarity of up to $99 \%$ with $p=0.00$. The threonine, serine and tyrosine residues are likely sites for modification by phosphorylation $^{38}$. According to predictive analysis (http://www.dabi. temple.edu/disphos/), the 43rd Thr residue is not a phosphorylation modification site with a $G$ allele at SNP556781 (Fig. S3b). The replacement of a solventexposed hydrophobic residue (Ile) with a hydrophilic residue (Thr) might impart thermostability to the protein structure $^{39}$. Therefore, this finding implies that the genotypes GG and AG at SNP556781 could enhance the stability of CHS relative to the genotype AA. However, tea plants with genotype AA have higher TCC values than those with genotypes GG and AG. In addition to amino residues, spatial conformation plays a critical role in the stability and activity of proteins. Further evaluations are warranted to explore the contributions of particular SNP mutations to the characteristics of proteins. Moreover, additional investigations of the metabolic mechanisms, especially upstream and downstream metabolites of catechins, are warranted and necessary to find genes that are closely related to $C H S$.

The regulation of traits can occur by a series of very complicated steps, and some are controlled by minor genes, with single genes that may possess multiple effects. As an illustration, $q \gamma 27$ in maize resulted from a $15.26 \mathrm{~kb}$ duplication at the $27-\mathrm{kDa} \gamma$-zein locus, which contains four genes. Genotypic results showed that this duplication significantly influenced the Lys/total levels and the expression levels of the four genes ${ }^{40}$. In accordance with these observations, the function of SNP556781 should be further investigated by an analysis that integrates other phenotypes. Furthermore, the construction of a coexpression network is a powerful strategy that can be employed for detecting the relationships among various genes and to gain a better understanding of the regulatory principles.

In the current study, the tea plants and the wild relatives used were native to the main tea production area in China, and only tea plants from Yunnan possessed the genotype AA. In other words, they included all three genotypes, indicating that there is rich genetic diversity in Yunnan. Consequently, it can be implied that the tea plant and its relatives in Yunnan preserve the most alleles as the most important reservoirs of genetic variation, which is in accordance with the results from Yao et al. ${ }^{3}$, Fang et al. ${ }^{41}$, and Huang et $\mathrm{al}^{42}$. The domestication effects may partially explain the higher frequency of the AA genotype, since TCC was negatively selected during domestication, in exchange for improved taste. TCC was affected by both genetic background and environment. Generally, TCC increases with higher temperature and stronger sunlight ${ }^{42}$. The TCC of tea plants decreased northward and 
eastward from the original location. Therefore, a decrease in the TCC is the result of both domestication and environmental effects. To effectively study the genetic mechanism and protection of tea plants, researchers should take full advantage of the existing natural resources for tea plants. Furthermore, additional resources are required to explore the exhaustive genotypic distribution in diverse species, since the majority of plants were $C$. sinensis var. sinensis in the current study.

No significant difference was found among the TCCs of the samples with the genotypes AG and GG. The TCC in tea plants with AA at SNP556781 was obviously higher than that observed in the plants with the genotypes AG and GG. It is likely that AA promotes the biosynthesis or inhibits the degradation of catechin and that its genotype-dependent capacity is genotype dependent. In conventional tea plants, flavonoid metabolism is enhanced under strong light, but the opposite results are observed in light-sensitive tea plants ${ }^{19}$. Catechins are usually decreased in green-leaf tea plants with shading treatment due to an alternation in the transcription level of the genes responsible for catechin biosynthesis ${ }^{43}$. In the present study, tea plants were grown in the same surroundings. Consequently, we concluded that the observed variation of DNA sequences, rather than the cultivation environment, is the contributing factor that influences catechin metabolism for these experimental plants. Moreover, not all the cultivars with the highest TCC values had the AA genotype, which suggests that other genes may also regulate TCC. A multi-environmental trial would further improve the validity of our findings, which deserves further analysis.

\section{Materials and methods Plant materials}

A controlled hybridization population with approximately 300 individuals was generated for this study. The male parent was 'Beiyue Danzhu' (BD, C. sinensis var. pubilimba), the landrace in Longzhou of Guangxi. The female parent selected for this study was 'Yingshuang' (YS, C. sinensis var. sinensis), which represents the national approved cultivar selected from natural hybrids between 'Fuding Dabaicha' (C. sinensis var. sinensis) and 'Yunnan Dayezhong' (C. sinensis var. assamica). Four offspring of the F1 population with relatively high or low TCC values named F1-1, F1-2, F1-3, and F1-4 were selected from the 'BD' $\times$ 'YS' population. The abovementioned tea plants and 170 other accessions that are representative of the core collection of tea germplasm ${ }^{27}$ (Tables S4 and S5) were planted and maintained through similar horticultural practices in the China National Germplasm Hangzhou Tea Repository located at the Tea Research Institute, Chinese Academy of Agricultural
Sciences. Three biological replicates were harvested at the "two leaves and a bud" stage in April 2018, and the samples were immediately flash frozen with liquid nitrogen and stored at $-80^{\circ} \mathrm{C}$ until further use.

\section{Catechin detection}

The samples were pretreated and examined as described in a previous study ${ }^{27}$. Specifically, the chromatographic peaks were identified by UV spectroscopy using a diode array detector, and the retention times were compared with those of the authentic standards.

\section{RNA and DNA extraction}

Total RNA was isolated using the RNAprep Pure Plant kit (Tiangen Biotech Co., Ltd., China) according to the manufacturer's instructions. The purity and degree of degradation of the RNA samples were examined by using a Nanodrop (Thermo Fisher Scientific, USA) and agarose gel electrophoresis, respectively. Then, the RNA samples were quantified using a Qubit RNAssay Kit (Qubit 2.0, USA), and the RNA integrity was assessed by using an Agilent RNA 6000 Pico Assay Kit (Agilent 2100, USA). Samples with RNA integrity values $>8.0$ along with a ratio of $1.9 \sim 2.1$ (A260/A280) and a ratio of $2.0 \sim 2.5$ (A260/A230) were selected for sequence analysis. Total DNA was isolated using a DNAsecure Plant Kit (Tiangen Biotech Co., Ltd., China) according to the manufacturer's instructions.

\section{Construction of a CDNA library and sequencing}

High-quality RNA samples from tea plants were prepared using an Illumina TruSeq RNA Sample Prep Kit (USA), and the cDNA libraries were constructed with an Ultra RNA Library Prep Kit for Illumina (USA). The cDNAs were purified using Beckman AMPure XP beads (USA) and subsequently assessed with an Agilent High Sensitivity DNA Kit (Agilent 2100, USA) for the detection of inserted cDNA fragments. Then, the cDNA libraries were quantified with a Bio-Rad KIT iQ SYBR Green kit (Bio-Rad CFX 96, USA) and subsequently sequenced using a TruSeq SBS Kit v3 (Illumina HiSeq2500, USA).

\section{Data filtering and mapping to the reference genome}

Clean reads were obtained after trimming and eliminating the adapter, ambiguous reads ('N') and low-quality reads (bases with Qphred $\leq 20$ more than $50 \%$ of total bases) with the FastX-toolkit based on Q30. The clean reads were then subsequently aligned to two reference genomes (http://pcsb.ahau.edu.cn:8080/CSS/ and http:// www.plantkingdomgdb.com/tea_tree/ $)^{29,30}$. An index of the reference genome was built using Bowtie v2.2.3, and paired-end clean reads were aligned to the reference genome using TopHat v2.0.12. A database of splice junctions was generated using TopHat based on the gene model annotation for an optimized mapping result. 


\section{Gene expression analyses}

The reads were mapped to each gene using HTSeq, and fragments per kilobase of exon model per million mapped reads (FPKM) were calculated on the basis of the length of the gene and read counts mapped to the reference genes. Differential expression analyses between two samples were performed based on negative binomial distribution through the DESeq $\mathrm{R}$ package (1.20.0). In addition, the $p$ values were adjusted by using Benjamini and Hochberg's approach. The differentially expressed genes (DEGs) were identified by DESeq, and the thresholds of the adjusted $p$ value and $\log 2$ (fold change) were 0.005 and 1 , respectively.

\section{Quantitative real-time PCR (qRT-PCR) analyses}

A total of 12 transcripts were randomly selected for validation of gene expression by using quantitative RT-PCR (qRT-PCR). The cDNAs were reverse transcribed using the PrimeScript ${ }^{\mathrm{TM}} \mathrm{RT}$ reagent qPCR kit (Tiangen Biotech Co., Ltd., China), and the qRT-PCR reactions were conducted using the following parameters: $95^{\circ} \mathrm{C}$ for $30 \mathrm{~s}$ and 40 cycles at $94{ }^{\circ} \mathrm{C}$ for $15 \mathrm{~s}$ and $60^{\circ} \mathrm{C}$ for $5 \mathrm{~s}$. Three independent biological replicates and three technical replicates of each reaction were performed using GAPDH as a reference gene. Fluorescence intensity was measured through the Applied Biosystems 7500 Sequence Detection System (Carlsbad, CA, USA), and the relative expression values of the genes were subsequently calculated based on the $2^{-\Delta \Delta \mathrm{Ct}}$ method.

\section{KEGG enrichment analysis of the DEGs}

The Kyoto Encyclopedia of Genes and Genomes (KEGG) enrichment analysis of the DEGs was implemented using KOBAS 2.0. The significance of the differential expression was assessed by relying on hypergeometry according to the following formula: $p=1-\sum_{i=0}^{m-1} \frac{\left(\begin{array}{c}M \\ i\end{array}\right)\left(\begin{array}{c}N-M \\ n-i\end{array}\right)}{\left(\begin{array}{c}N \\ n\end{array}\right)}$. In this formula, $n, M$, and $m$ represent the number of DEGs, genes in one pathway, and DEGs in one pathway, respectively. The DEGs were substantially enriched in the KEGG pathway with a false discovery rate of $<0.05$.

\section{SNP validation}

The DEGs were aligned to the genes that were responsible for flavonoid metabolism. The matched DEGs that had an FPKM >1000 were used for SNP screening, and 28 SNPs in the coding regions of the genes were selected for functional investigation. These SNPs were tested in 176 tea accessions by using KASP, and 20 accessions were randomly selected for two replicate analyses. The SNPs and primers that were used for KASP are displayed in Table S1. The tea accessions were grouped based on SNP types, and their catechin contents were compared using analysis of variance (ANOVA) at a threshold of $p<0.05$. The results were subsequently analyzed and visualized by using R 3.5.1.

\section{Acknowledgements}

This work was supported by the Earmarked Fund for China Agriculture Research System (CARS-019), the Chinese Academy of Agricultural Sciences through the Agricultural Science and Technology Innovation Program (CAASASTIP-2017- TRICAAS) to L.C., and the National Natural Science Foundation of China (31500568) to J.Q.M.

\section{Author details}

${ }^{1}$ Key Laboratory of Tea Biology and Resources Utilization, Ministry of Agriculture and Rural Affairs, Tea Research Institute of the Chinese Academy of Agricultural Sciences, 9 South Meiling Road, Hangzhou, Zhejiang 310008, China. ${ }^{2}$ College of Horticulture and Forestry Science, Huazhong Agricultural University, 1 Shizishan Street, Hongshan District, Wuhan, Hubei 430070, China. ${ }^{3}$ Tea Research Institute, Yunnan Academy of Agricultural Sciences, Menghai, Yunnan 666201, China

\section{Author contributions}

Conceived and designed the experiments: Liang Chen, Chen-Kai Jiang, JianQiang Ma; conducted the experiments: Chen-Kai Jiang, Yu-Fei Liu; analyzed the data: Chen-Kai Jiang, Jie-Dan Chen; wrote the paper: Chen-Kai Jiang; revised the paper: Liang Chen, De-Jiang Ni.

\section{Conflict of interest}

The authors declare that they have no conflict of interest.

Supplementary Information accompanies this paper at (https://doi.org/ 10.1038/s41438-020-0247-y).

Received: 10 October 2019 Revised: 23 December 2019 Accepted: 4 January 2020

Published online: 01 March 2020

\section{References}

1. Chen, L., Yu, F. L. \& Yang, Y. J. Germplasm and Genetic Improvement of Tea Plant. 28-35 (China Agricultural Science and Technology Press, Beijing, 2006).

2. Xia, E. H. et al. Tea plant information archive: a comprehensive genomics and bioinformatics platform for tea plant. Plant Biotechnol. J. https://doi.org/ 10.1111/pbi.13111, (2019).

3. Yao, M. Z., Ma, C. L., Qiao, T. T., Jin, J. Q. \& Chen, L. Diversity distribution and population structure of tea germplasms in China revealed by EST-SSR markers. Tree Genet. Genomes 8, 205-220 (2012).

4. Chen, L., Zhou, Z. X. \& Yang, Y. J. Genetic improvement and breeding of tea plant (Camellia sinensis) in China: from individual selection to hybridization and molecular breeding. Euphytica 154, 239-248 (2007).

5. Chen, L., Apostolides, Z. \& Chen, Z. M. Global Tea Breeding: Achievements, Challenges and Perspectives 38 (Springer-Zhejiang University Press, Hangzhou, 2012).

6. Zhu, J., Wen, D., Yu, Y., Meudt, H. M. \& Nakhleh, L. Bayesian inference of phylogenetic networks from bi-allelic genetic markers. PLoS Comput. Biol. 14, e1005932 (2018).

7. Palumbo, F., Qi, P., Pinto, V. B. \& Barcaccia, K. M. D. A. Construction of the first SNP-based linkage map using genotyping-by-sequencing and mapping of the male-sterility gene in leaf chicory. Front. Plant Sci. 10, 276 (2019).

8. Huang, X. H. et al. Genomic architecture of heterosis for yield traits in rice. Nature 537, 629 (2016).

9. Chagné, D. et al. Validation of SNP markers for fruit quality and disease resistance loci in apple (Malus $\times$ domestica Borkh.) using the OpenArray ${ }^{\circledR}$ platform. Hortic. Res. 6, 30 (2019).

10. Lander, E. S. The new genomics: global views of biology. Science 274, 536 (1996).

11. Niu, S. et al. Genetic diversity, linkage disequilibrium, and population structure analysis of the tea plant (Camellia sinensis) from an origin center, Guizhou 
plateau, using genome-wide SNPs developed by genotyping-by-sequencing. BMC Plant Biol. 19, 328 (2019).

12. $\mathrm{XU}, \mathrm{L}$. Y. et al. High-density SNP linkage map construction and QTL mapping for flavonoid-related traits in a tea plant (Camellia sinensis) using 2b-RAD sequencing. BMC Genomics. 19, 955 (2018).

13. Zhu, J. Y. et al. Global dissection of alternative splicing uncovers transcriptional diversity in tissues and associates with the flavonoid pathway in tea plant (Camellia sinensis). BMC Plant Biol. 18, 266 (2018).

14. Magdalena, K. C., Klaudia, S., Iwona, K. \& Rafał, B. Expression of the flavonoid pathway genes in carrot plants tolerant to salt stress. N. Biotech. 33, S166 (2016).

15. Bálintová, M., Brunakova, K., Petijova, L. \& Cellárová, E. Targeted metabolomic profiling reveals interspecific variation in the genus Hypericum in response to biotic elicitors. Plant Physiol. Biochem. 135, 348-358 (2019).

16. Zhang, Z. Z. et al. Antioxidant properties and mechanisms of tea polyphenols. Nut Sci. Tech. 3259, 131-159 (2008).

17. Guo, F., Guo, Y. F., Wang, P., Wang, Y. \& Ni, D. J. Transcriptional profiling of catechins biosynthesis genes during tea plant leaf development. Planta 246, 1139-1152 (2017).

18. $\mathrm{Xu}, \mathrm{Y}$. Q. et al. Quantitative analyses of the bitterness and astringency of catechins from green tea. Food Chem. 258, 16-24 (2018).

19. Feng, Z. Q., Liu, M. Y. \& Ruan, J. Y. Metabolomics analysis reveals the metabolic and functional roles of flavonoids in light-sensitive tea leaves. BMC Plant Biol. 17, 64 (2017)

20. Pheomphun, P., Treesubsuntorn, C. \& Thiravetyan, P. Effect of exogenous catechin on alleviating $\mathrm{O}_{3}$ stress: the role of catechin-quinone in lipid peroxidation, salicylic acid, chlorophyll content, and antioxidant enzymes of Zamioculcas zamiifolia. Ecotox. Environ. Safe. 180, 374-383 (2019).

21. Quan, J. J. et al. Green tea extract catechin improves cardiac function in pediatric cardiomyopathy patients with diastolic dysfunction. J. Biomed. Sci. 26, 32 (2019).

22. Farzaei, M. H., Bahramsoltani, R., Abbasabadi, Z., Braidy, N. \& Nabavi, S. M. Role of green tea catechins in prevention of age-related cognitive decline: pharmacological targets and clinical perspective. J. Cell. Physiol. 234, 2447-2459 (2019).

23. Ahmad, R. S. et al. Preventive role of green tea catechins from obesity and related disorders especially hypercholesterolemia and hyperglycemia. J. Transl. Med. 13, 79 (2015).

24. Wang, W. Z. et al. An insight into catechins metabolic pathways of Camellia sinensis based on genome and transcriptome analysis. J. Agric. Food Chem. 66, 4281-4293 (2018).

25. Gui, X. L. et al. Dynamic changes in catechin levels and catechin biosynthesisrelated gene expression in albino tea plants (Camellia sinensis L.). Plant Physiol. Biochem. 71, 132-143 (2013).

26. Caesar-Johnson, S. J. et al. Comprehensive characterization of cancer driver genes and mutations. Cell 173, 371 (2018).
27. Jin, J. Q., Ma, J. Q., Ma, C. L., Yao, M. Z. \& Chen, L. Determination of catechin content in representative Chinese tea germplasms. J. Agric. Food Chem. 62, 9436-9441 (2014).

28. Cao, K. et al. Comparative population genomics identified genomic regions and candidate genes associated with fruit domestication traits in peach. Plant Biotechnol. J. https://doi.org/10.1111/pbi.13112, (2019).

29. Wei, C. L. et al. Draft genome sequence of Camellia sinensis var. sinensis provides insights into the evolution of the tea genome and tea quality. Proc. Natl Acad. Sci. USA 115, E4151-E4158 (2018).

30. Xia, E. H. et al. The tea tree genome provides insights into tea flavor and independent evolution of caffeine biosynthesis. Mol. Plant. 10, 866-877 (2017).

31. Yu, F. L. Discussion on the origin and center of tea plant. J. Tea Sci. 6, 1-8 (1986).

32. Ma, J. Q. et al. Large-Scale SNP discovery and genotyping for constructing a high-density genetic map of tea plant using specific-locus amplified fragment sequencing (SLAF-seq). PLOS ONE 10, e.0128798 (2015).

33. Koech, R. K. et al. Identification of novel QTL for black tea quality traits and drought tolerance in tea plants (Camellia sinensis). Tree Genet. Genomes 14, 9 (2018).

34. Koech, R. K. et al. Functional annotation of putative QTL associated with black tea quality and drought tolerance traits. Sci. Rep. 9, 1465 (2019).

35. Liu, S. G. et al. Characterization of genome-wide genetic variations between two varieties of tea plant (Camellia sinensis) and development of InDel markers for genetic research. BMC Genomics. 20, 935 (2019).

36. Brenda, W. S. Flavonoid biosynthesis. A colorful model for genetics, biochemistry, cell biology and biotechnology. Plant Physiol. 126, 485-493 (2001).

37. Zhang, X. B., Abrahan, C., Colquhoun, T. A. \& Liu, C. J. A Proteolytic regulator controlling chalcone synthase stability and flavonoid biosynthesis in. Arabidopsis. Plant Cell. 29, 1157-1174 (2017).

38. Soufi, B., Soares, N. C., Ravikumar, V. \& Macek, B. Proteomics reveals evidence of cross-talk between protein modifications in bacteria: focus on acetylation and phosphorylation. Curr. Opin. Microbiol. 15, 357-363 (2012).

39. Khurana, J., Singh, R. \& Kaur, J. Engineering of Bacillus lipase by directed evolution for enhanced thermal stability: effect of isoleucine to threonine mutation at protein surface. Mol. Biol. Rep. 38, 2919-2926 (2011).

40. Deng, $M$. et al. The genetic architecture of amino acids dissection by association and linkage analysis in maize. Plant Biotechnol. J. 15, 1250-1263 (2017).

41. Fang, W., Cheng, H., Duan, Y., Jiang, X. \& Li, X. Genetic diversity and relationship of clonal tea (Camellia sinensis) cultivars in China as revealed by SSR markers. Plant Syst. Evol. 298, 469-483 (2012).

42. Huang, Y. et al. Cenozoic plant diversity of Yunnan: a review. Plant Divers. 6 271-282 (2016)

43. Liu, L. L. et al. Metabolite profiling and transcriptomic analyses reveal an essential role of UVR8-mediated signal transduction pathway in regulating flavonoid biosynthesis in tea plants (Camellia sinensis) in response to shading. BMC Plant Biol. 18, 233 (2018). 